\title{
Applications of Cone Beam Computerized Tomography in Orthodontics: A Review
}

\author{
Anuraj Singh Kochhar ${ }^{1}$, Gurkeerat Singh ${ }^{2 *}$ and Harshita Gupta ${ }^{3}$ \\ ${ }^{1}$ Former Consultant Orthodontist, Max Hospital Gurgaon, Haryana, India \\ ${ }^{2}$ Vice Principal, Professor and Head, Department of orthodontics and dentofacial orthopaedics, Sudha Rustagi College of Dental Sciences and Research, Haryana, \\ India
}

${ }^{3}$ Post-graduate Resident, Department of orthodontics and dentofacial orthopaedics, Sudha Rustagi College of Dental Sciences and Research, Haryana, India

*Corresponding author: Gurkeerat Singh, Vice Principal, Professor and Head, Department of orthodontics and dentofacial orthopaedics, Sudha Rustagi College of Dental Sciences and Research, Faridabad, Haryana, India.
Received Date: August 19, 2020

Published Date: September 07, 2020

\begin{abstract}
Cone Beam Computerized Tomography (CBCT) is being comprehensively used for diagnosis and treatment planning in orthodontics worldwide. Thorough visualization of craniofacial structures with accuracy and precision gives CBCT an upper hand over its apposite like 3D CT systems (fan beam) and 2D imaging systems. Moreover, it minimizes patient discomfort, improves patient contentment and extensively helps the orthodontist with a better understanding of treatment outcome by incorporating artificial intelligence along with traditional imaging. It thereby facilitates an orthodontist with a complete guide throughout treatment phases. In this article, we aim to review various applications of cone beam computerized tomography (CBCT) in the field of orthodontics with patients scanned for routine orthodontic treatment.
\end{abstract}

Keywords: CBCT; Orthodontic applications; Orthodontic diagnosis; 3D Imaging system

Abbreviations: CBCT: Cone Beam Computerized Tomography; 3D: Three-Dimensional; CT: Computerised Tomography; 2D: Two-Dimensional; mSv: Millisievert; HU: Hounsfield Unit; OSA: Obstructive Sleep Apnoea; TMJ: Temporo-mandibular Joint; MRI: Magnetic Resonant Imaging; STL: Stereolithography; FEM: Finite Element Model

\section{Introduction}

With the evolution of cephalometric radiography in 1931 by Broadbent in the United States and Hofrath in Germany [1]. cephalometry has been used very extensively. These cephalometric radiographs are heavily relied on by orthodontists today to measure angular and linear dimensions using various anatomic landmarks. For years, these three-dimensional (3D) landmarks have been diagnosed and treatment planned based on twodimensional (2D) imaging [2]. Localization of these points can be difficult because of superimposing anatomic structures and differential magnification of bilateral structures which results in image distortion. The use of 3D computed tomography avoids anatomic superimposition as well as problems caused by magnification and thereby permits evaluation of the craniofacial structures with less distortion than plane film views [3]. But the drawbacks of such 3D CT systems (fan beam) include high cost, large physical size, and the relatively high radiograph dosages

(approximately $2000 \mathrm{mSv}$ ) which limits its use in craniofacial diagnosis. Although CBCT equipment has existed over the past decade it has become possible to produce clinical systems that are both inexpensive and small enough to be used in craniofacial diagnosis in recent times. For orthodontic diagnosis and treatment planning cone beam computerized tomography has become an integral part of orthodontic practice as it provides with extensive visualization of craniofacial structures [4]. CBCT essentially reduces the scan time, thus reducing the effective radiation to the patient. This technology provides increased precision, lower doses and lower costs as compared to medical fan-beam computerized tomography (Figure 1) [5,6]. CBCT provides three-dimensional images that facilitate the transition of orthodontic imaging from initial diagnosis to image guidance throughout the treatment phase [7]. 
In this article, we aim to review various applications of cone beam computerized tomography (CBCT) in the field of orthodontics keeping all the advantages in mind.

\section{Advantages of CBCT as Compared to CT Scans $[5,8,9]$}

(Table 1)

Table 1

\begin{tabular}{|c|c|}
\hline Variable Field of View & Collimation of x-rays to the area of interest minimizes unnecessary exposure and irradiation. \\
\hline Dose Reduction & Ranges from $29-477 \mu$ Sv 10,11 and can be further reduced up to $40 \%$ by changing patient's position. \\
\hline Fast Scan Time & Scanning speed is high $(5,8.9$ or 26.9-seconds scan time) reducing motion artefacts and fewer images for reconstruction. \\
\hline Image Accuracy & $\begin{array}{c}\text { The processed image is of high resolution as it is measured in voxels instead of pixels and often is sharper than a conventional } \\
\text { CT. }\end{array}$ \\
\hline Unique Display Mode & Ability to perform multiplanar reformatting \\
\hline
\end{tabular}

\section{Applications of CBCT in Orthodontics}

CBCT generated panoramic $x$-rays: CBCT panoramic x-rays generate exact duplication of the dentition in 1:1 form with exact angulation and root curvature which is not possible in conventional panoramic radiographs where in elongation of root and faulty angulation are usually encountered (Figure 2). The panoramic views of СВCT also gives exact location of teeth with the bone coverage as there is facility by the software to calculate the distance and amount of bone coverage in HU units (Figure 3).

CBCT generated lateral cephalograms: As there is exact duplication of bone morphology the cephalograms from CBCT machine are exact and precise in there measurement with advantage of viewing both right and left side (in a single scan). The superimposition of bone structure is not present and cephalometric landmarks which are otherwise difficult to trace in routine cephalograms are easily located making diagnostic analysis more accurate and precise (Figure 4). In a study, there was no statistical difference noted between CBCT measurements and direct craniometric measurements making them the most potential diagnostic tool [12].

3D Reconstruction: The slices in CBCT are very thin as compared to the conventional medical CT $(.125-.300 \mathrm{~mm})$ which gives an accurate 3D reconstruction [13]. No measurable difference in accuracy was noted from 3D scans with the usage of multiple projection images for reconstruction in a study by April et al [14]. These reconstructions are useful in viewing the bone morphology, especially in bony asymmetry and congenital anomalies like cleft lip and palate. The reconstruction can be done suitably as per the operator's choice to view the deeper structures so as to evaluate the extent and area of bony deformity (Figure 5). These 3D reconstructions can be used for simulation in orthognathic surgical planning such as osteotomies, distraction osteogenesis, etc. $[9,15,16]$.

CBCT in cleft lip and palate: Patients with cleft lip and palate have skeletal malformation that are very difficult to assess on routine cephalograms and panoramic views. With use of CBCT the exact extent of cleft can be determined i.e.-

1. whether it is affecting the deeper structures or not.

2. impacted teeth in line of cleft (Figure 6).

3. the degree of maxillary arch collapse.

4. to measure the amount of expansion already done.

5. the amount of bone that is to be grafted in cleft region can be easily calculated $[17,18]$ (Figure 7).

Figure 1

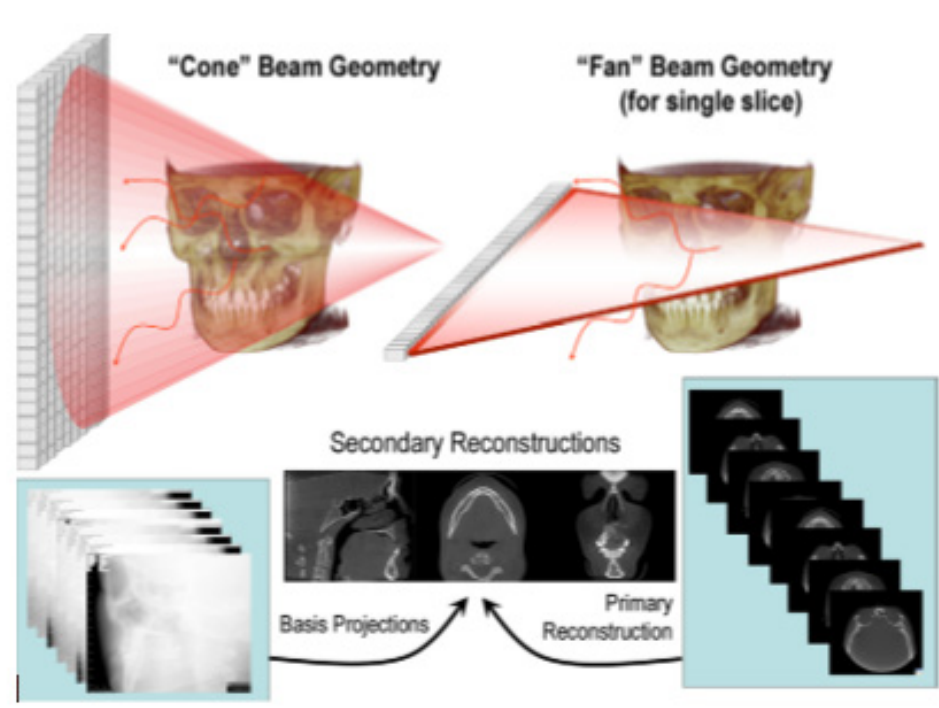




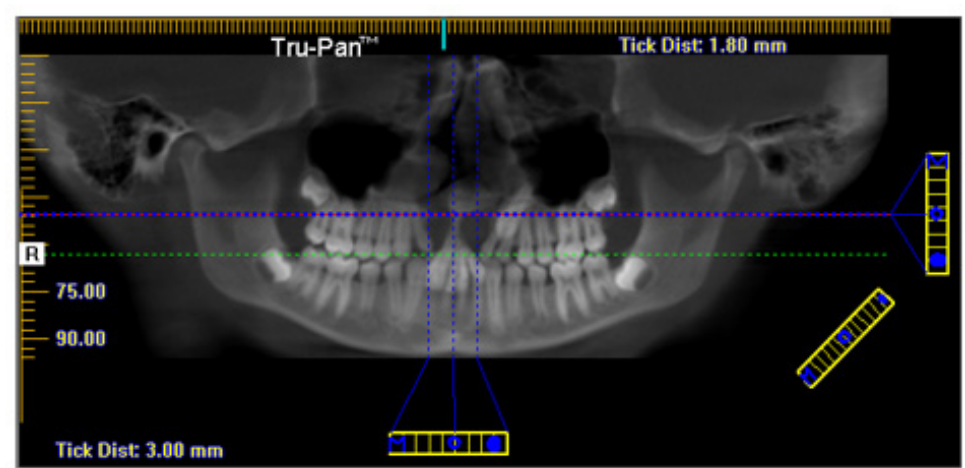

Figure 2

Figure 3
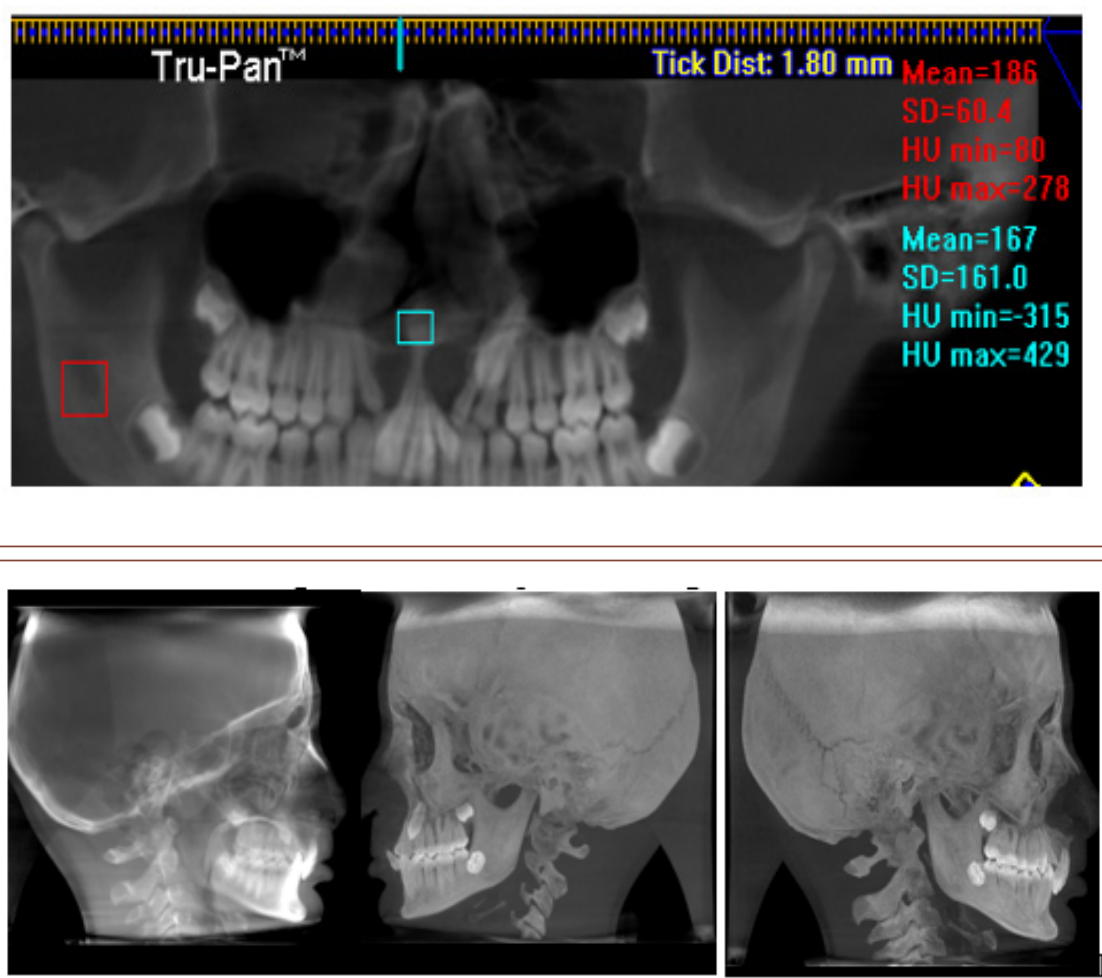

Figure 4

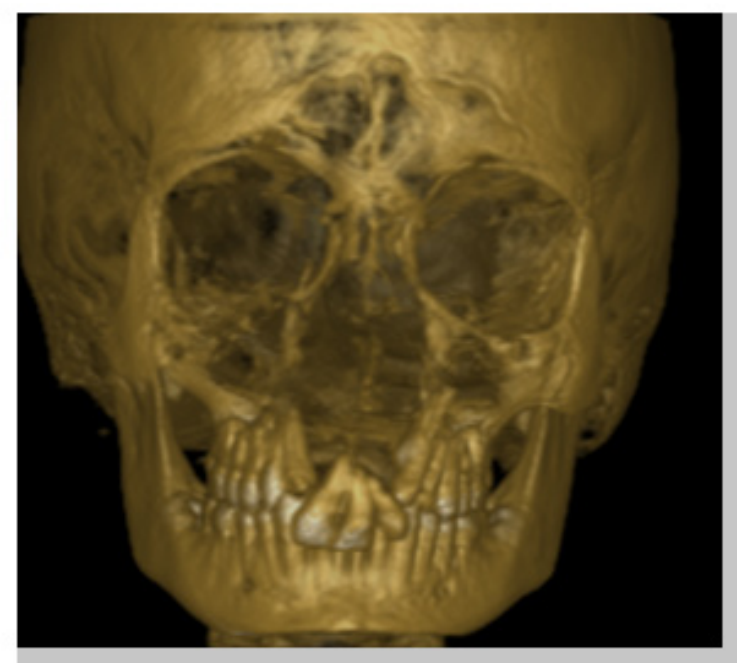

Figure 5 


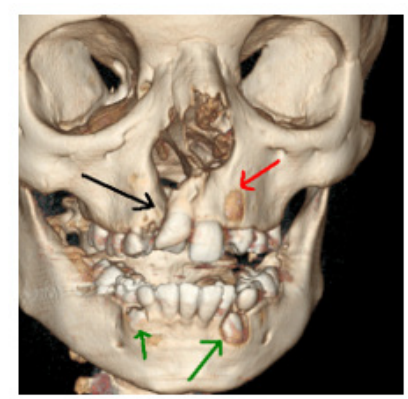

Figure 6: Black arrow showing the cleft, red showing the impacted canine, red showing erupting canine.

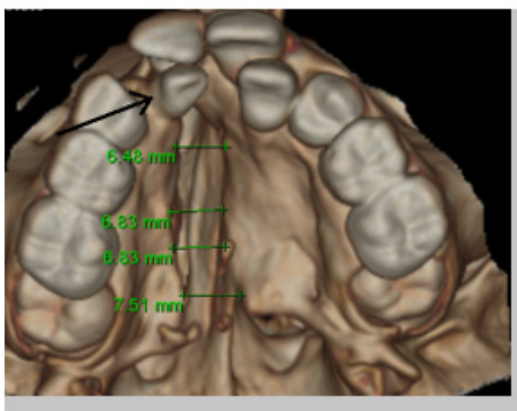

Figure 7: Black arrow showing lingual placed lateral incisor, green reading showing exact cleft dimension anterior posteriorly.

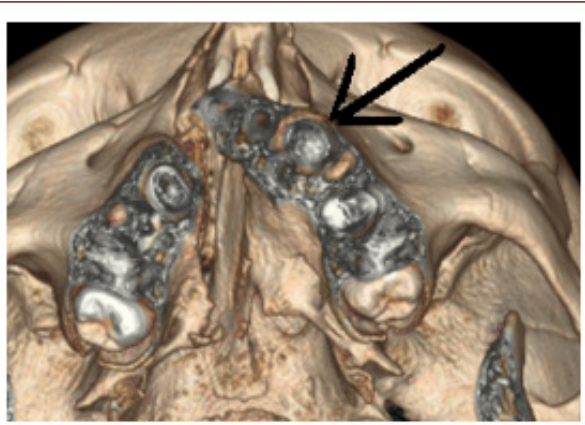

Figure 8: Arrow indicating emergence of impacted canine on axial slice.

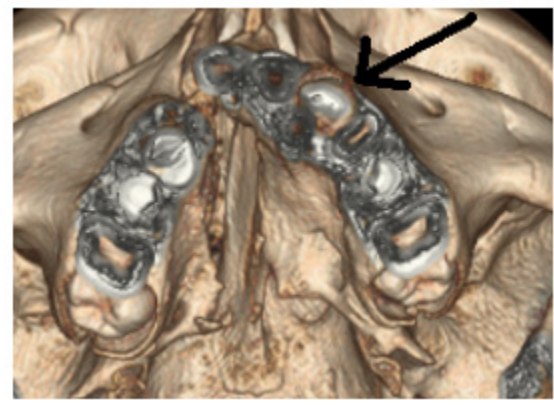

Figure 9: Impacted canine clearly visible on next slice view.

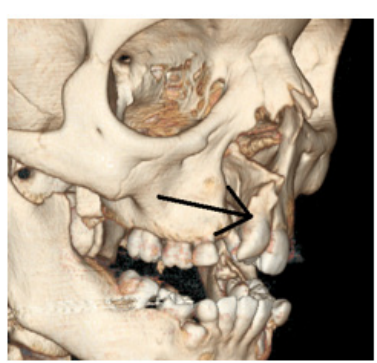

Figure 10: Arrow showing no bone coverage on distal surface of canine. 
CBCT in impacted and transposed canines: Impacted canine pose quite a challenge to proper treatment planning in orthodontics. The routine investigation requires a minimum of $4 \mathrm{x}$-rays to diagnose the position of the impacted canine. But even after using the shift switch technique the position of maxillary canine sometimes remains doubtful. CBCT imaging is precise in determining not only the labial/lingual relationship but also a more exact angulation and depth of the impacted canine (Figure 8-10). Precise and exact location from the alveolar bone can be calculated and planned accordingly to expose the impacted canine as stated by Leah Walker et al [19].

CBCT in facial asymmetries: With comprehensive visualization and image accuracy, CBCT provides quantitative measurement and comparison between right and left sides of the craniofacial structures, resulting in detailed diagnosis and management of facial asymmetries. Both hard and soft tissue facial asymmetry can be diagnosed and precisely quantified. Although in the gonial region, Rahime et al stated to use 2D imaging as well for detailed diagnosis of asymmetry in that region [18]. Metzger et al demonstrated the use of CBCT to simulate and plan orthognathic surgeries for facial asymmetry [20].

CBCT in obstructive sleep apnoea (OSA) and enlarged adenoids: 3D and volumetric analysis of airway dimensions in patients suffering from obstructive sleep apnoea or enlarged adenoids gives better understanding of treatment planning which was lacking with 2D diagnostic tools. The bony fusions of cervical vertebrae have been found in related to various craniofacial syndromes and may lead to OSA. To diagnose these fusions with 2D imaging is cumbersome and results in false measurements in airway analysis. CBCT helps in sufficient understanding of different morphologies of cervical vertebrae and helps in better airway analysis when compared to 2D imaging [21].

CBCT for TMJ assessment: CBCT can be used in the assessment of TMJ anatomy, condylar position, developmental anomalies, in the diagnosis of various types of arthritis, and evaluation of internal derangement. Addition to this CBCT can also assess condylar fractures, ankyloses, cysts and tumours $[9,22]$

CBCT for alveolar boundary conditions and other jaw pathologies: Compromised alveolar boundary conditions before treatment may lead to various complications during and post treatment. Other occult jaw pathologies like sinus blockage, cyst, tumor, calcified plaque, root resorption, fractures, etc are sometimes obsolete to judge and diagnose with 2D imaging systems [23]. In such cases CBCT should be used with careful considerations.

CBCT and artificial intelligence: CBCT scan in STL format produces 3D software models for an orthodontist to diagnose and plan the treatment without the hassle of using alginate impressions. This will result in reducing chairside time, patient comfortability and comprehensive details of anatomic structures. These digital models can be used for various finite element (FEM) studies as well as in the execution of treatment processes such as clear aligner therapy, indirect bonding procedures like insignia ${ }^{\mathrm{TM}}$ and in other customized brackets and wires. 3D metal printing of appliances too can be advocated with the help of these study models [24, 25].

CBCT and mini-implants: CBCT allows accurate mini-implant placement with proper guidance of bone density and quality, interradicular relationships and visualization of important anatomic structures which may be harmed while placement [25-28]

\section{Conclusion}

CBCT offers a 3D image that can be used to aid in orthodontic diagnosis, treatment planning in all three planes of space. The use of CBCT data shortly will change the way records are taken and treatment is rendered. Craniofacial CBCT analysis is an excellent method for quantifying and analyzing surface and deep craniofacial structures.

\section{Acknowledgment}

None.

\section{Conflict of Interest}

No conflict of interest.

\section{References}

1. Rakosi $T$ (1982) Cephalometry and radiographic analysis. In: Carruthers GB, editor. An atlas and manual of cephalometric radiography. Wolfe Medical Publications Ltd, London p: 7.

2. Hechler SL (2008) Cone-beam CT: applications in orthodontics. Dent Clin North Am 52(4): 809-823.

3. Papadopoulos MA, Jannowitz C, Boettcher P, Henke J, Stolla R, et al. (2005) Three-dimensional fetal cephalometry: an evaluation of the reliability of cephalometric measurements based on three-dimensional CT reconstructions and on dry skulls of sheep fetuses. J Craniomaxillofac Surg 33(4): 229-237.

4. Agrawal JM, Agrawal MS, Nanjannawar LG, Parushetti AD (2013) CBCT in orthodontics: the wave of future. J Contemp Dent Pract 14(1): 153157.

5. Farman AG, Scarfe WC (2009) The basics of maxillofacial cone beam computed tomography. Seminars in Orthodontics 15(1): 2-13.

6. Schulze D, Heiland M, Thurmann H, Adam G (2004) Radiation exposure during midfacial imaging using 4-and 16-slice computed tomography, cone beam computed tomography systems and conventional radiography. Dentomaxillofacial Radiology. 33(2): 83-86.

7. William E Harrell Jr (2009) 3D Diagnosis and Treatment Planning in Orthodontics. Semin Orthod 15: 35-41.

8. Venkatesh E, Elluru SV (2017) Cone beam computed tomography: basics and applications in dentistry. J Istanb Univ Fac Dent 51(3 Suppl 1): S102-S121.

9. Marothiya S, Patel V, Jain D, Agrawal S, Joshi S, et al. (2019) Cone beam computed tomography in orthodontics: A literature review. International Journal of Applied Dental Sciences 5(2): 233-240.

10. Ludlow JB, Davies-Ludlow LE, Mol A (2007) Dosimetry of recently introduced CBCT units for oral and maxillofacial radiology. In Proceedings of the $16^{\text {th }}$ international congress of dento maxillofacial radiology, Beijing, China 26(30): 97.

11. Schulze D, Heiland M, Thurmann H, Adam G (2004) Radiation exposure during midfacial imaging using 4-and 16-slice computed tomography, 
cone beam computed tomography systems and conventional radiography. Dentomaxillofacial Radiology. 33(2): 83-86.

12. Gribel BF, Gribel MN, Frazão DC, McNamara Jr JA, Manzi FR (2011) Accuracy and reliability of craniometric measurements on lateral cephalometry and 3D measurements on CBCT scans. Angle Orthod 81(1): 26-35.

13. Kim M, Huh KH, Yi WJ, Heo MS, Lee SS, et al. (2012) Evaluation of accuracy of 3D reconstruction images using multi-detector CT and conebeam CT. Imaging Sci Dent 42(1): 25-33.

14. Brown AA, Scarfe WC, Scheetz JP, Silveira AM, Farman AG (2009) Linear accuracy of cone beam CT derived 3D images. Angle Orthod 1;79(1): 150-157.

15. Cevidanes LHS, Bailey LJ, Tucker GR, Styner MA, Mol A, et al. (2005) Superimposition of 3D cone-beam CT models of orthognathic surgery patients. Dentomaxillofac Radiol 34(6): 369-375

16. Bamgbose BO, Adeyemo WL, Ladeinde AL, Ogunlewe MO (2008) Conebeam computed tomography (CBCT): the new vista in oral and maxillofacial imaging. Nig Q J Hosp Med 18(1): 32-35.

17. Wortche R, Hassfeld S, Lux CJ, Mussig E, Hensley FW, et al. (2006) Clinical application of cone beam digital volume tomography in children with cleft lip and palate. Dentomaxillofacial Radiology 35(2): 88-94.

18. Kuijpers MA, Pazera A, Admiraal RJ, Bergé SJ, Vissink A, et al. (2014) Incidental findings on cone beam computed tomography scans in cleft lip and palate patients. Clin Oral Investig 18(4): 1237-1244.

19. Walker L, Enciso R, Mah J (2005) Three-dimensional localization of maxillary canines with cone-beam computed tomography. Am J Orthod Dentofacial Orthop 128(4): 418-423.

20. Nur RB, Çakan DG, Arun T (2016) Evaluation of facial hard and soft tissue asymmetry using cone-beam computed tomography. Am J Orthod Dentofacial Orthop 149(2): 225-237.

21. Metzger MC, Hohlweg-Majert B, Schwarz U, Teschner M, Hammer B, et al. (2008) Manufacturing splints for orthognathic surgery using a threedimensional printer. Oral Surg Oral Med Oral Pathol Oral Radiol Endod 105(2): e1-7.

22. Sonnesen L, Jensen KE, Petersson AR, Petri N, Berg S, et al. (2013) Cervical vertebral column morphology in patients with obstructive sleep apnoea assessed using lateral cephalograms and cone beam CT. A comparative study. Dentomaxillofacial Radiology 42(6): 20130060.

23. Różyło-Kalinowska I (2019) Cone Beam Computed Tomography (CBCT) in TMJ Imaging. In Imaging of the Temporomandibular Joint, Springer, Cham Pp: 125-131.

24. Dale Miles A (2009) Interpreting the Cone Beam Data Volume for Occult Pathology. Semin Orthod 15(1): 70-76.

25. Thakkar D, Ghosh A, Keshwani T (2020) Digital Workflow for CBCTGuided Customized Miniscrew-Assisted Rapid Palatal Expansion (3D Digital MARPE): A Clinical Innovation. Journal of Indian Orthodontic Society 0301574220926690.

26. Srinivasan S (2019) Reliability and Accuracy of Assessing TAD-Tooth Root Contact using CBCT (Doctoral dissertation, The Ohio State University), USA.

27. Cassetta M, Sofan AA, Altieri F, Barbato E (2013) Evaluation of alveolar cortical bone thickness and density for orthodontic mini-implant placement. J Clin Exp Dent 5(5): e245-52.

28. Eigenwillig P, Ludwig B, Bumann A (2020) Safe and Precise TAD Placement in the Anterior Palate with Simple and Inexpensive TAD Guides. Temporary Anchorage Devices in Clinical Orthodontics 6: 577586. 